\title{
Micromechanics analysis of particulate-reinforced composites and their failure mechanisms
}

\author{
ZHIFA DONG, YONGLI WU \\ Institute of Mechanics, Chinese Academy of Sciences, Beijing, 100080, \\ People's Republic of China
}

Stress fields and failure mechanisms have been investigated in composites with particles either surface treated or untreated under uniaxial tension. Previous experimental observation of failure mechanisms in a composite with untreated particles showed that tensile cracks occurred mostly at the polar region of the particle and grew into interfacial debonding. In a composite with surface-treated particles, however, shear yielding and shear cracking proceeded along the interphase-matrix interface at the polar area of the matrix and thus may improve the mechanical behaviour of the material. The finite element calculations showed that octahedral shear stress at the polar and longitudinal areas of the particle treated by coupling agents is much larger than that of materials with untreated particles, and the shear stress distribution around the interface is sensitive to the interphase property. The results suggest that a three-phase model can describe the composites with surface-treated fillers.

\section{Introduction}

In a composite, adding second-phase particles to plastics to modify mechanical properties is a common practice. Many authors have proved that adding rigid particles to thermoplastics can enhance the elastic modulus substantially [1-4], but the mechanical behaviour of composites reinforced with particles greatly depends on the applied stress fields and the response of the matrix, particle and particle-matrix interface. Lu Sinien et al. [5] showed that the following two facts have an important effect on the final physical properties of composites: (1) a strengthening effect which is due to deformation of the matrix surrounding the particles being constrained by inclusions; (2) a weakening effect caused by filler-incurred damage. This particle-induced damage can be affected by many factors, such as shape, size and volume fraction of particles, and the mechanical properties of the particles and matrix, etc. Among these factors, interfacial debonding is definitely the most significant one. To improve the interfacial debonding strength, the surface of the particles was usually treated with coupling agents. This suggests a three-phase particle-reinforced composite. The proposed material will be a poly-blend of spherical high modulus inclusions encapsulated within a uniform layer of low modulus elastomer (interphase) whose thickness depends on the chemistry and processing conditions of the particles, and is dispersed throughout the matrix.

In this paper, a three-dimensional finite element method was used to analyse the stress distributions around the particle and the interphase to investigate the cause of different failure mechanisms for the com- posite with particles either surface treated or untreated. The distributions were studied in the interior of the three-phase composites under uniaxial tension.

\section{Failure processes observed in previous experiments}

According to experimental evidence $[5,6]$, failure always begins in the polar region of the inclusion both with treated and untreated beads when a specimen is subjected to a tensile load. Failure mechanism around the particles whose surface has been treated with coupling agents is that, as was observed in the experiments, failure occurs first in the matrix, beginning at one of the poles in the same direction as the tensile force; the interfacial debonding and cracking processes in plastic deformation of the matrix can be observed in some local microareas, and the fillers are completely covered by the polymer (in untreated fillers they are bare). As the load increases, the yielding zone gradually extends and the matrix in the polar region of the particle gradually debonds from the particles. Growing along the tensile direction, voids appear in the polar zone. When the load reaches the macroscopic yield strength of the material, some large deformations in the matrix are produced in the area between the glass beads. Finally, a shear yielding forms and the material loses its load-carrying capacity. Due to strong interfacial adhesion, the interfacial debonding and cracking processes in the tensile test occur gradually.

In the reference material with untreated inclusions, however, the matrix was liable to form tensile cracks 
during tensile deformation at the polar region of the particles where debondings have taken place, even though the matrix does not yield. As the load increases the debondings expand rapidly. At a much lower stress level the particle is liable to completely or partly debond from the matrix, and a large void forms at the polar region of the particles.

There may be two explanations for the different failure mechanisms. One possibility is that stress fields were different around particles whose surface was treated or untreated by coupling agents in the interior of the composite - this is investigated in this paper. Another possibility is that there were different adhesion strengths for different coupling agents at the interface between the particles and the matrix. The mechanism has been studied for a series of materials with different coupling agents to obtain different interfacial adhesions [5].

Owing to the addition of a coupling agent, it is reasonable to assume that the inclusions are coated

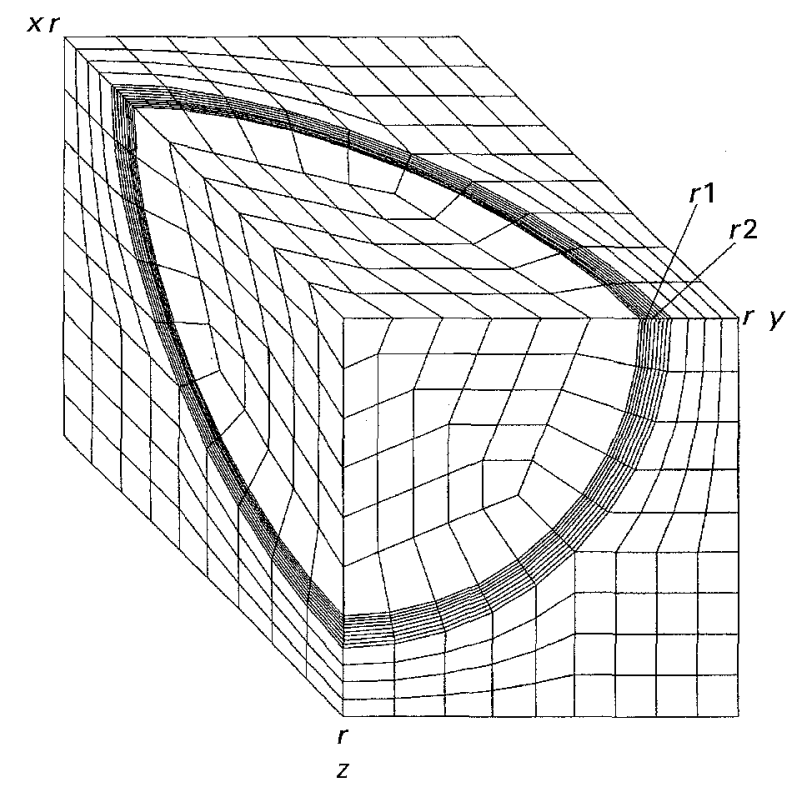

Figure I A three-dimensional finite element model.

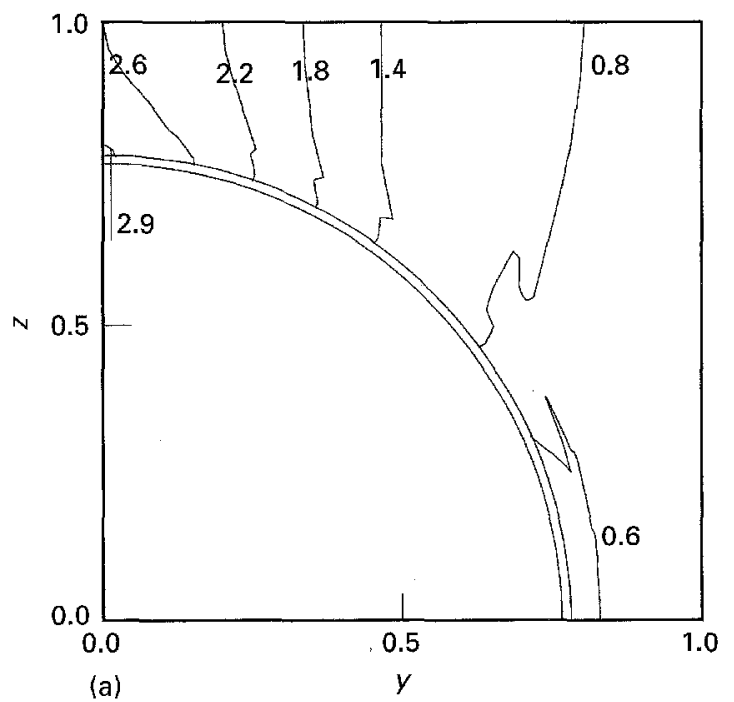

with a thin layer of low modulus elastomer (interphase) and dispersed in the matrix. The different stress fields around the particles treated or untreated by a coupling agent will be studied by means of the finite element method (FEM) in the following section.

\section{Finite element analysis}

It is assumed that the spherical inclusions packed in a cubic way are embedded in a composite. A threedimensional finite element model is shown in Fig. 1. The finite element model represents only one-eighth of the composite volume element, which is reasonable on the basis of the geometric symmetry. Perfect interfacial couplings between the particle and the matrix, or between particles, interphase and matrix, were also assumed. The well known finite element program package ADINA was used to study the elastic stress fields around the interface of the particle as well as in the interior of the composite. The boundary conditions and solution procedures used follow those of Agarwal and Broutman [7]. The unit model is subjected to a uniaxial tension in the $z$-direction and no tractions in the $x$ - or $y$-directions.

Young's modulus and Poisson's ratio were assumed to be: $35 \mathrm{GPa}$ and 0.23 for the particle, $1.0 \mathrm{GPa}$ and 0.40 for the matrix, and $0.05 \mathrm{GPa}$ and 0.48 for the interphase. These values are approximate to the experimental values reported by $\mathrm{Lu}$ Sinien et al. [5].

\subsection{Stress distributions around the particles}

Due to the geometric symmetry of the unit cell, only the stresses on the $x=0$ plane were chosen for analysis. They are represented as a ratio $\sigma / \bar{\sigma}_{z}$, where $\bar{\sigma}_{z}$ is the average stress applied to the composite. Thus, the ratio represents the stress concentration around the particle.

Fig. 2a and $b$ show the major principal stresses around the particle with or without an interphase, respectively. The stress distributions of the composite with an interphase are significantly different from the

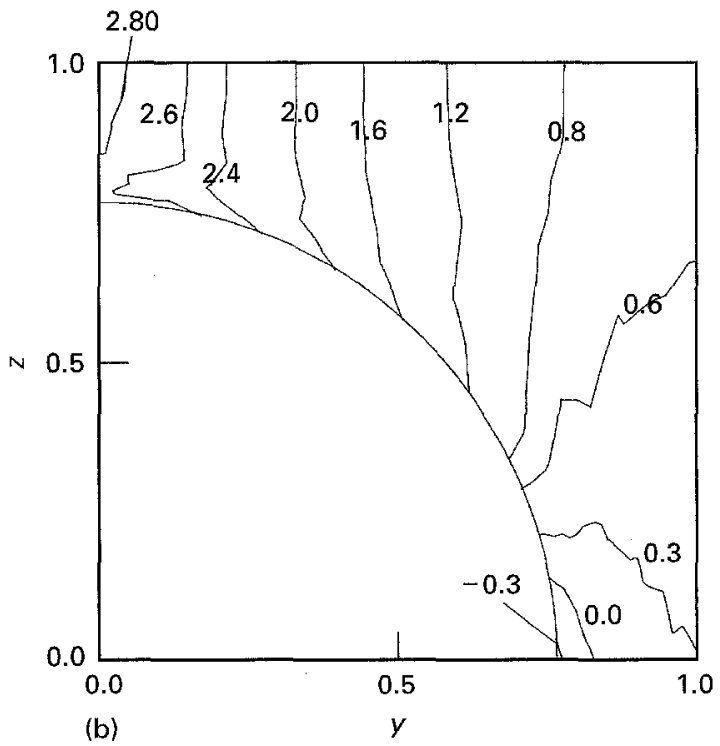

Figure 2 The major principal stress distributions around the particle with (a) and without (b) interphase for $V_{i}=23.56 \%, V_{\mathrm{i}}=\pi / 6\left(r^{1} / r\right)^{3}$. 
material without a coating. For a composite with an interphase, the maximum major principal stress occurs in the polar region, just at the interface between the interphase and matrix, but in the reference material with untreated inclusions, this maximum stress lies on the matrix above the polar region. In the longitudinal region, the maximum compressive stress occurs at the interface in the reference material, and in the composite with an interphase the major principal stresses are always tensile stresses. Fig. 3 shows the principal stresses at the interphase-matrix interface. The stresses have been compared with the results of the composite without an interphase. The calculations indicate that the tensile stresses would be responsible for the tensile microcracking and the debonding at the polar region of the particle.

Octahedral shear stresses, $\tau_{\text {oct }}$, were distributed around the inclusion as shown in Fig. 4, where $\tau_{\text {oct }}$ is

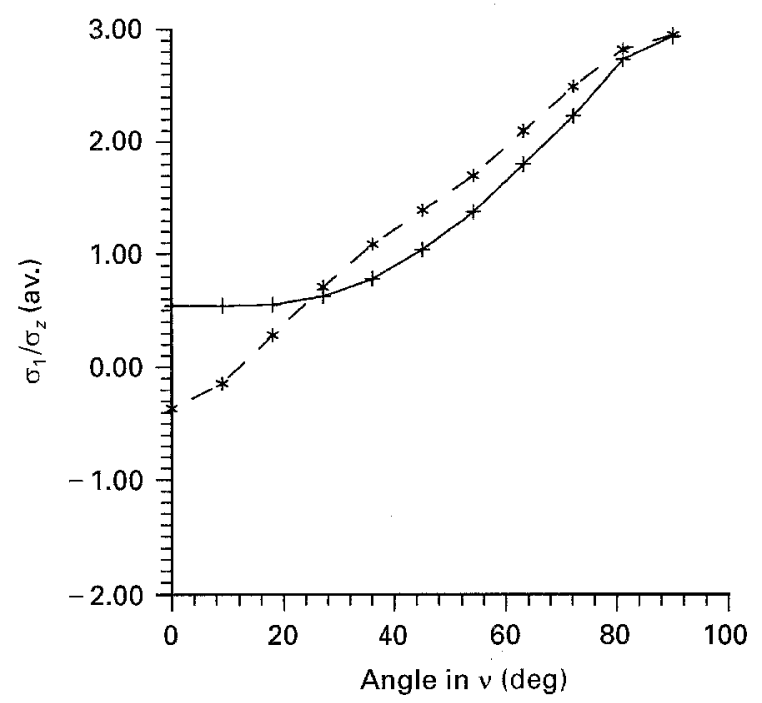

Figure 3 Major principal stresses in the matrix along the interface shown in Fig. $5 .++$, With an interphase; $*-*$, Without an interphase.

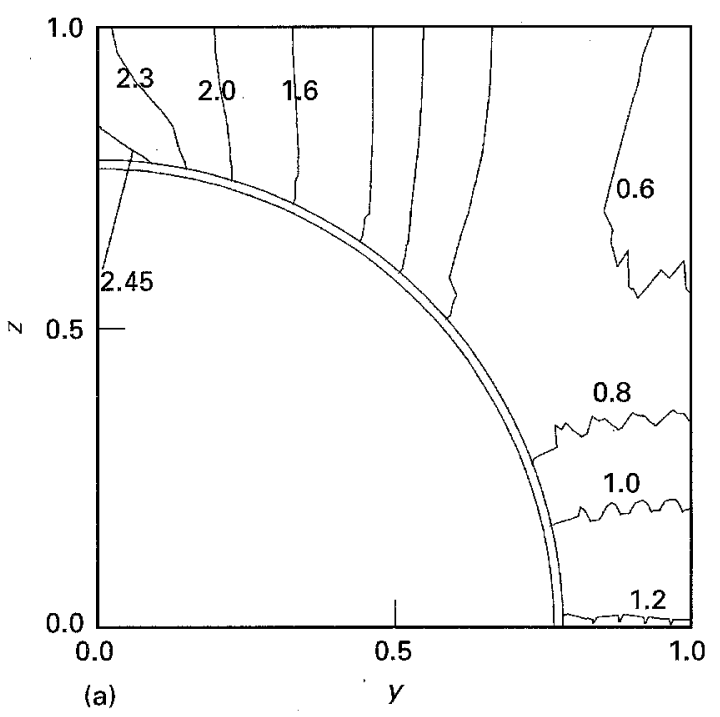

defined as:

$$
\tau_{\mathrm{oct}}=\frac{1}{3}\left[\left(\sigma_{1}-\sigma_{2}\right)^{2}+\left(\sigma_{2}-\sigma_{3}\right)^{2}+\left(\sigma_{3}-\sigma_{1}\right)^{2}\right]^{1 / 2}
$$

where $\sigma_{1}, \sigma_{2}$ and $\sigma_{3}$ are principal stresses at a certain point in the composite. $\tau_{\text {oct }}$ is normalized with respect to the stress $[(\sqrt{6}) / 2] \bar{\sigma}_{z}$ and the value of $\tau_{\text {oct }}$ is equal to the equivalent stress: Adding the interphase into the particulate-reinforced composite has greatly changed the internal shear stress distributions of the material. Looking at the $\tau_{\text {oct }}$ distributions along the interface, shown in Fig. 5, the maximum octahedral shear stresses in the three-phase composite are much larger than those in the material without an interphase, and are located in the polar and longitudinal regions, respectively. On the other hand, the maximum octahedral shear stress at the interface of the two-phase composite is within the $18^{\circ}<\theta<35^{\circ}$ region. The differences of the shear stress distributions at the interface may have an effect on the formation of shear yielding and the growth of interfacial debonding around the interface.

The calculations may present a reasonable interpretation for the different failure mechanisms of particulate-reinforced composites whose particles were surface treated or not. The coating or interphase can greatly change the stress fields around the particles. Although the debond crack has a similar tensile stress concentration at the polar region of the particle, the concentration of the shear stresses within this region at the interface for the three-phase composite is definitely larger than that for the reference material. This corresponds qualitatively to the experimental results of section 2 , in that debonding appears mostly under a much lower stress level at the polar region of the particle in the reference material.

\subsection{Effects of interphase property and aspect ratio}

Fig. 6 shows the major principal stress $\sigma_{1}$ and the octahedral shear stress, $\tau_{\text {oct }}$, distributions around the

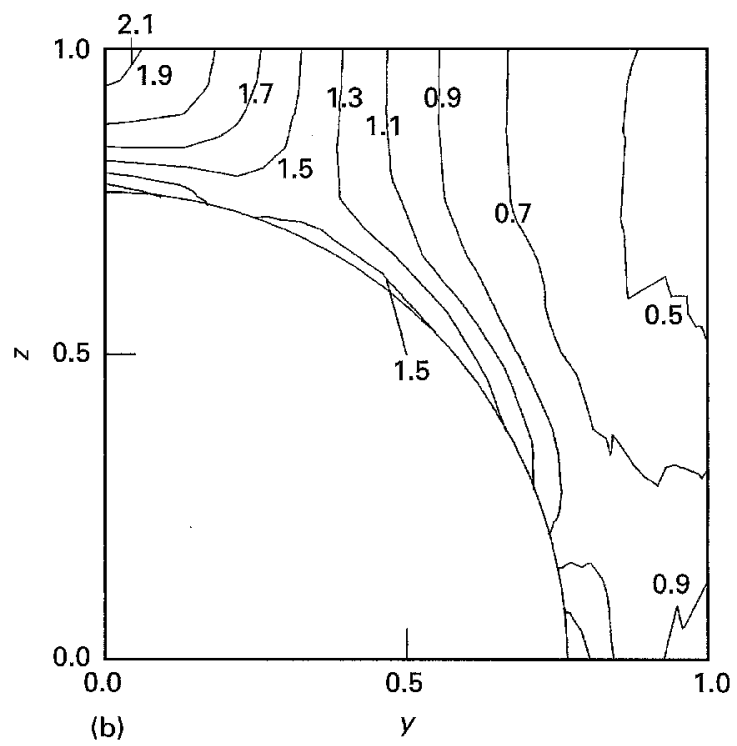

Figure 4 Octahedral shear stress, $\tau_{\mathrm{oct}}$, distributions around the particle with (a) and without (b) interphase for $V_{\mathrm{i}}=23.56 \%$. 


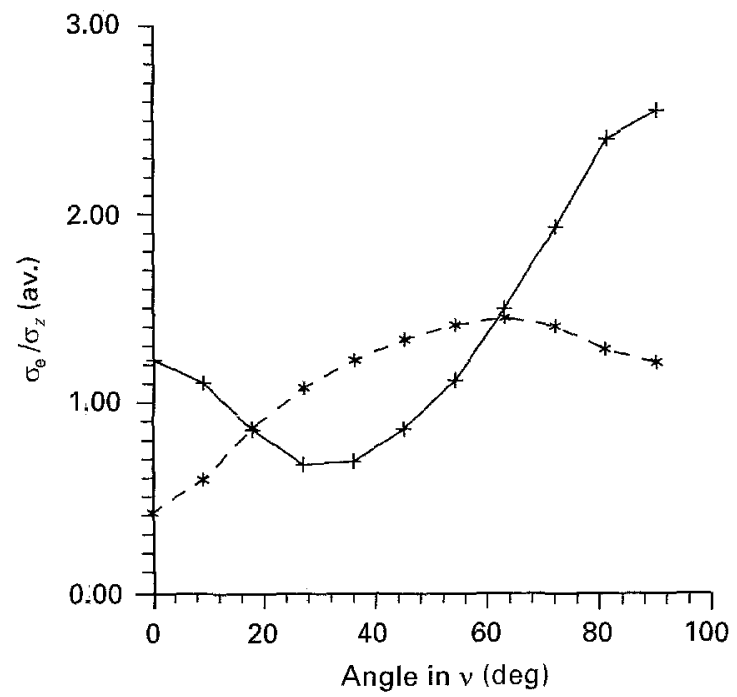

Figure 5 Matrix octahedral shear stresses $\tau_{\text {oct }}$, along the interface shown in Fig. $7 .++$, With an interphase; $*-*$, Without an interphase.

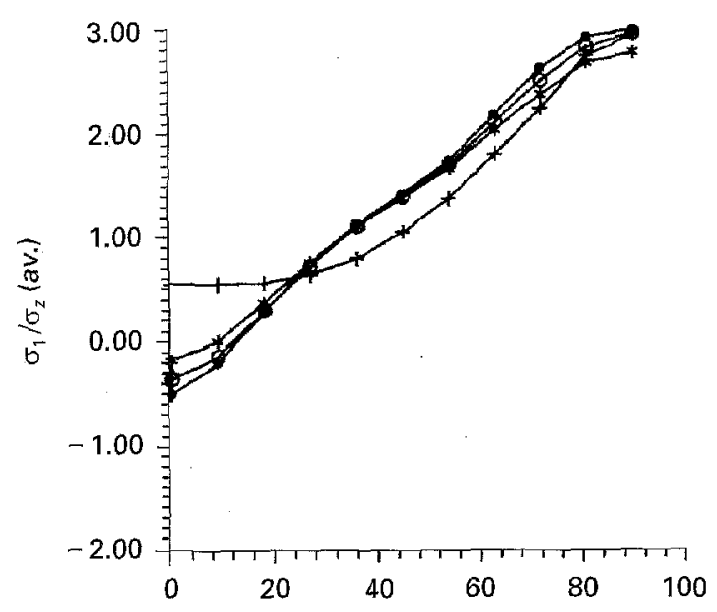

(a) Angle in $v(\mathrm{deg})$

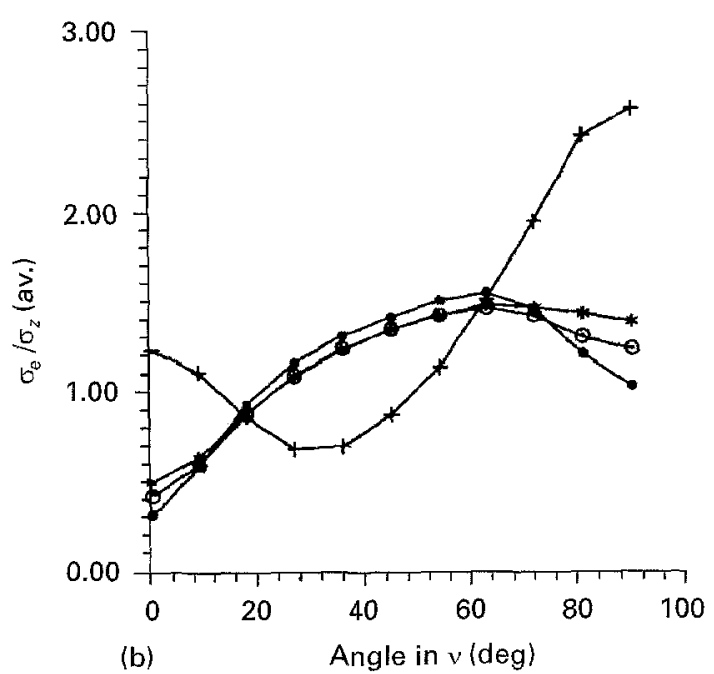

Figure $\sigma$ Interphase property, $E_{\mathrm{f}}$ (interphase) $/ E_{\mathrm{m}}$ (matrix), dependence of $\sigma_{1}$ (a) and $\tau_{\text {oct }}$ (b) along the interface. $E_{\mathrm{f}} / E_{\mathrm{m}}:++, 0.05$; $*-*, 0.50 ;-\mathrm{O}-\mathrm{O}-1.00 ; \rightarrow-\mathbf{-}, 35.00$.

interface between the interphase and the matrix for various Young's moduli of the interphase. The major principal stresses have a similar tensile stress concentration around the interface, but the octahedral shear stress distributions are greatly dependent on the inter-

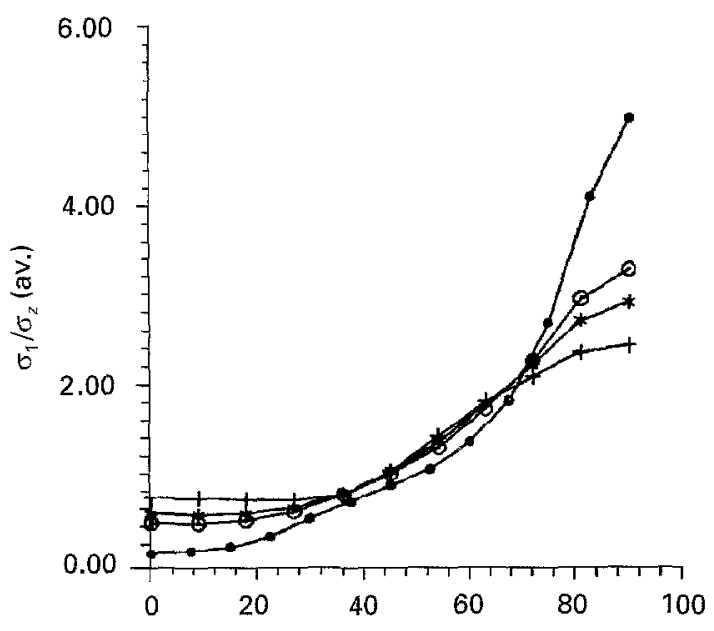

(a) Angle in $v$ (deg)

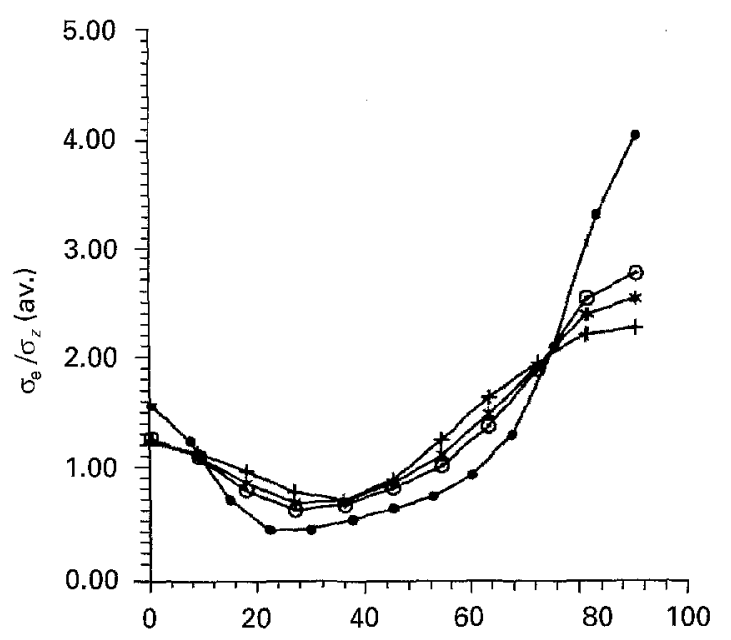

(b) Angle in $v$ (deg)

Figure 7 Volume fraction of particles, $V_{\mathrm{i}}$, dependence of $\sigma_{1}(\mathrm{a})$ and $\tau_{\text {oct }}$ (b) along the interface. $V_{\mathrm{i}}(\%):++, 10 ; *-*, 23.56 ;-\mathrm{O}-\mathrm{O}-, 30$; -- 45 .

phase properties. In particular, the shear stresses at the polar and longitudinal regions increase with decreasing Young's moduli of the interphase, and indicate that shear yielding and shear cracking occur preferentially in the matrix around the polar and longitudinal regions for a softer interphase material.

Fig. 7 shows $\sigma_{1}$ and $\tau_{\text {oct }}$ distributions for various volume fractions of inclusions. $\sigma_{1}$ increases extensively with an increase in volume fraction of inclusions at the polar region, which assumes that, in this region, tensile debonding is most likely to occur. On the other hand, $\tau_{\mathrm{oct}}$ slightly increases with an increase in volume fraction of inclusions.

This is reasonable in that although the high concentration of tensile stresses for composites with high volume fractions of surface-treated particles is more likely to cause interface debondings, a much higher shear stress than that of composites without an interphase at the debonding area may yield the matrix to resist the debonding spread and may improve the mechanical behaviour of the two-phase composite.

\section{Conclusions}

Stress fields and failure mechanisms have been investigated in a composite with or without an interphase. 
1. The calculations show that, for a composite without an interphase, tensile cracks are more likely to occur at the polar region of the particle and grow into interfacial debondings. The situation is different in the composite with surface-treated particles, where matrix shear yielding near the interphase-matrix interface at the polar area of particles may cause interfacial shear cracking and thus may improve the mechanical behaviour of the two-phase material. The matrix octahedral shear stress at the polar area and longitudinal area of particles treated by coupling agents is much larger than that of the material without an interphase. Concentration of the major principal stress at the polar area of the interface increases more rapidly than that of the shear stress as the volume fraction of the particle increases.

2. The calculations agree qualitatively with the failure mechanisms observed in the experiments. The results suggest that for a composite with surface-treated fillers, a three-phase model can describe its failure mechanisms and its interior stress distributions.

\section{Acknowledgements}

This work was supported by the National Natural Science Foundation of China and Laboratory for Nonlinear Mechanics of Continuous Media, Institute of Mechanics, Chinese Academy of Sciences.

\section{References}

1. J. LAHILI and A. PAUL, J. Mater. Sci. 20 (1985) 2253.

2. U. YILMAZER and R. J. FARRIS, Polym. Comps. 4 (1983) 1.

3. L. NICOLAIS, G. GUERRA, C. MIGLIARESI, L. NICOdEMO and A. T. DiBenedetto, Composites 12 (1981) 33.

4. M. E. J. DEK KERS and D. HEIKENS, J. Appl. Polym. Sci. 28 (1983) 3809.

5. LU SINIEN, YAN LIN, ZHU XIAOGUANG and QI ZONGNENG, J. Mater. Sci. 27 (1992) 4633.

6. M. PEgORARO, F. SEVERINI and L. DI LANDRO, Polym. Comps. 9 (1988) 252.

7. B. D. AGARWAL and L. J. BROUTMAN, Fibre Sci. Technol. 7 (1974) 63.

Received 4 October 1994

and accepted 13 February 1996 